\title{
Compared Efficiencies of Conversions between Charge and Spin Current by Spin-Orbit Interactions in Two- and Three-Dimensional Systems
}

\author{
J.-C. Rojas-Sánchez ${ }^{1, *}$ and A. Fert ${ }^{2, \dagger}$ \\ ${ }^{1}$ Université de Lorraine, CNRS UMR 7198, Institut Jean Lamour, F-54011 Nancy, France \\ ${ }^{2}$ Unité Mixte de Physique, CNRS, Thales, Université Paris-Sud, Université Paris-Saclay, 91767 Palaiseau, France
}

(Received 14 March 2019; published 17 May 2019)

\begin{abstract}
Conversions between charge and spin currents by spin-orbit interactions have become usual in today's spintronics, either by using the spin Hall effect (SHE) and inverse spin Hall effect (ISHE) of heavy metals or the Edelstein effect and inverse Edelstein effect of a two-dimensional (2D) electron gas. These conversions can be characterized by the spin Hall angle for SHE or ISHE and by the conversion parameters $q_{\text {IC }}$ for EE and $\lambda_{\text {IEE }}$ for IEE. Using typical experimental values of these different parameters for heavy metals and 2D electrons of topological insulators (TIs), we show that, compared to heavy metals, the TIs lead to a gain of about an order of magnitude in the conversion between spin to charge or charge to spin currents and even more in the production of voltage or electrical power by conversion of spin currents. Thus, we anticipate a giant spin Seebeck power generated in devices designed with an insulating magnetic layer and 2DEGs of a TI as $\alpha$-Sn or Rashba 2DEGs. We also discuss the detrimental role of additional shunts in these conversion experiments.
\end{abstract}

DOI: 10.1103/PhysRevApplied.11.054049

\section{INTRODUCTION}

The conversions between charge and spin currents are essential operations in any spintronic device. In classical spintronics, spin filtering by magnetic materials is used for these conversions. Typically, in a spin-transfer torque random access memories [1,2] for example, tunneling from a reference ferromagnetic layer creates the spin-polarized current used to switch the magnetization of the free layer by spin transfer torque, which means charge to spin current conversion followed by transfer of spin momentum to nonvolatile magnetization. For the inverse conversion, the tunneling magnetoresistance transforms the spin information into charge current and voltage signals. More efficient conversions between charge and spin are obtained today by using spin-orbit coupling (SOC) effects. Charge currents can be converted into pure spin currents by the spin Hall effect (SHE) of heavy nonmagnetic metals (HM) as Pt or W; see the review by Hoffmann [3]. Similar conversions can be obtained by the Edelstein effect (EE) associated with the locking between the spin and charge degrees of freedom in a bidimensional electron gas (2DEG) at a Rashba interface or a topological insulator (TI) surface or interface [4-13]. Inversely, conversions from spin to charge current can be obtained with the inverse SHE (ISHE) and inverse EE (IEE) [4-15].

\footnotetext{
*juan-carlos.rojas-sanchez@univ-lorraine.fr

†albert.fert@cnrs-thales.fr
}

\section{PARAMETERIZATION OF SPIN-TO-CHARGE CONVERSIONS}

Comparing the conversion efficiencies of SHE (or ISHE) and EE (or IEE) is not straightforward: the conversion by SHE or ISHE involves three-dimensional (3D) current densities in 3D conductors (heavy-metal and adjacent layers). In contrast, the conversion by EE or IEE involves two-dimensional (2D) current densities in 2DEGs and 3D current densities in 3D adjacent layers. In a layer with 3D conduction states (the heavy-metal layer), the equivalent of a 2D current density is the total current in a layer of unit width, which corresponds to the integral of the 3D current density from the top to the bottom of the layer. In this paper, we show how spin-to-charge conversion in $3 \mathrm{D}$ and $2 \mathrm{D}$ systems can be compared and we illustrate our results on typical examples of experimental results.

We begin with a reminder of the usual expression of the spin-to-charge conversion by SHE, ISHE, EE, and ISHE. Our notation is $j_{c}^{3 \mathrm{D}}$ and $j_{s}^{3 \mathrm{D}}$ (defined as the difference between charge current with opposite spin, $j_{s}^{3 \mathrm{D}}=j_{c+}^{3 \mathrm{D}}-$ $j_{c-}^{3 \mathrm{D}}$ ) for respectively charge and spin 3D current densities (in $\mathrm{A} / \mathrm{m}^{2}$ ) and $j_{c}^{2 \mathrm{D}}$ and $j_{s}^{2 \mathrm{D}}$ (defined as $j_{s}^{2 \mathrm{D}}=j_{c+}^{2 \mathrm{D}}-$ $j_{c-}^{2 \mathrm{D}}$ ) for respectively charge or spin $2 \mathrm{D}$ current densities (in $\mathrm{A} / \mathrm{m}$ ).

The conversions by SHE or ISHE between 3D spin and charge current are characterized by a number, the spin Hall angle defined as the ratio between the transverse spin 
current density $j_{s}^{3 \mathrm{D}}$ and the longitudinal charge current density $j_{c}^{3 \mathrm{D}}$ in an infinite material:

$$
\theta_{\mathrm{SHE}}=j_{s}^{3 \mathrm{D}} / j_{c}^{3 \mathrm{D}}
$$

The conversions by EE or IEE are conversions between a 2D charge current density $j_{c}^{2 D}$ in a $2 \mathrm{DEG}$ and a $3 \mathrm{D}$ spin current density $j_{s}^{3 \mathrm{D}}$ ejected into or injected from an adjacent layer. They are characterized by the conversion coefficients $\lambda_{\text {IEE }}$ for IEE (notation introduced in Refs. $[9,16]$ and $q_{\text {ICS }}$ for EE (notation introduced in Ref. [12]) as follows:

$$
\begin{aligned}
& j_{c}^{2 \mathrm{D}} / j_{s}^{3 \mathrm{D}}=\lambda_{\mathrm{IEE}}, \\
& j_{s}^{3 \mathrm{D}} / j_{c}^{2 \mathrm{D}}=q_{\mathrm{ICS}} .
\end{aligned}
$$

In measurements of spin-to-charge conversion, the spin current $j_{s}^{3 \mathrm{D}}$ seen by the experimentalist is defined in different ways in different types of experiments. The situation is simpler in spin to charge experiments by ISHE or IEE, as, for example, in an experiment of spin pumping by ferromagnetic resonance (FMR) from a ferromagnetic layer (FM) into a heavy metal or 2DEG.If there is no spin relaxation in an intermediate layer or by interface spin memory loss, generally the linewidth FMR broadening directly indicates the spin current density $j_{s}$ received and accommodated by the heavy-metal layer at its interface $(z=0)$ or by the 2DEG and relaxing inside the heavy-metal layer down to its free interface at $z=t(t=$ thickness) or in the $2 \mathrm{DEG}$. Thus the conversion is due to purely internal mechanisms. In the heavy metal, the conversion depends only on its internal parameters $\theta_{\mathrm{SHE}}$, spin diffusion length $\lambda_{\mathrm{SF}}$, and thickness $t$ [3]. In the 2DEG, the coefficient $\lambda_{\text {IEE }}$ of spin-to-charge conversion by IEE depends only on intrinsic parameters of the 2DEG [16]. We see that the situation is slightly different in most experiments of charge-to-spin conversion in which it is more difficult to separate internal parameters of the SOC system from external ones, as, for example, interface parameters [16].

\section{SPIN-TO-CHARGE CURRENT CONVERSION: INVERSE SPIN HALL EFFECT VS INVERSE EDELSTEIN EFFECT}

We thus begin with the calculation of the conversion coefficients in the simpler situation of spin-to-charge conversion by ISHE and IEE. In addition, to begin with the very simplest case, we consider that the magnetic material is insulating [such as $\mathrm{Y}_{3} \mathrm{Fe}_{5} \mathrm{O}_{12}$, yttrium iron garnet (YIG), for example], which suppresses any shunting by a conducting material other than the heavy metal or the 2DEG. We treat the shunting effects at the end of the paper.

In ISHE and from classical spin-transport equations, the spin current density decaying as a function of the depth $z$ from $z=0$ to $z=t$ can be written as [3,17-19]

$$
j_{s}^{3 \mathrm{D}}(z)=\sinh \left[(z-t) / \lambda_{\mathrm{SF}}\right] / \sinh \left[t / \lambda_{\mathrm{SF}}\right] \times j_{s}^{3 \mathrm{D}},
$$

where $j_{s}^{3 \mathrm{D}}$ is the spin current density received at the interface $z=0, t$ the layer thickness, and $\lambda_{\mathrm{SF}}$ the spin diffusion length. The $3 \mathrm{D}$ density of the resulting charge current is

$$
j_{c}^{3 \mathrm{D}}(z)=\theta_{\mathrm{SHE}} \sinh \left[(z-t) / \lambda_{\mathrm{SF}}\right] / \sinh \left[t / \lambda_{\mathrm{SF}}\right] \times j_{s}^{3 \mathrm{D}} .
$$

The equivalent 2D current density $j_{c}^{2 \mathrm{D}}$ (total current in a unit width layer) is obtained by integration from $z=0$ to $z=t$ in Eq. (5), which leads to

$$
j_{c}^{2 \mathrm{D}} / j_{s}^{3 \mathrm{D}}=\theta_{\mathrm{SHE}} \lambda_{\mathrm{SF}} \tanh \left[t / 2 \lambda_{\mathrm{SF}}\right] .
$$

The maximum conversion, $\left[j_{c}^{2 \mathrm{D}} / j_{s}^{3 \mathrm{D}}\right]_{\max }=\theta_{\mathrm{SHA}} \lambda_{\mathrm{SF}}$, is obtained for $t \gg \lambda_{\mathrm{SF}}$ and the efficiency of the conversion is characterized by the length $\lambda_{\text {ISHE }}^{*}$ as follows:

$$
\lambda_{\mathrm{ISHE}}^{*}=\theta_{\mathrm{SHE}} \lambda_{\mathrm{SF}}
$$

To compare the efficiency of ISHE and IEE, $\lambda_{\text {ISHE }}^{*}$ has to be compared to the IEE length $\lambda_{\text {IEE }}$ defined by Eq. (2) and characterizing the spin-to-charge conversion by the inverse Edelstein effect at Rashba or topological interfaces. The gain $g$ is given by

$$
g=\frac{\lambda_{\mathrm{IEE}}}{\lambda_{\mathrm{ISHE}}^{*}}=\frac{\lambda_{\mathrm{IEE}}}{\theta_{\mathrm{SHE}} \lambda_{\mathrm{SF}}} .
$$

In Table I, we present experimental values of $\lambda_{\text {IEE }}$ for the topological insulator and Rashba 2D states, as well as experimental values of $\theta_{\mathrm{SHE}}, \lambda_{\mathrm{SF}}$, and corresponding values of $\lambda_{\text {ISHE }}^{*}$ in Pt, $\mathrm{W}$, and Ta (among many experimental data, we choose typical examples in a well-admitted range for the spin Hall angle and spin diffusion length of these classical heavy metals). We compare the conversion efficiencies of systems for which these data have been obtained at room temperature: Pt, W, and Ta for ISHE and the TI $\alpha$-Sn for IEE. In the last column of Table I, the ratio $g=\lambda_{\text {IEE }} / \lambda_{\text {ISHE }}^{*}$ represents the gain $g$ in production of the charge current $j_{c}^{2 \mathrm{D}}$ for the same injected spin current density $j_{s}^{3 \mathrm{D}}$ brought by a TI such as $\alpha$-Sn as a replacement for the heavy metal. These gains vary between 6.2 (gain from the ISHE of $\mathrm{W}$ to the IEE of $\alpha$-Sn) and 11 (gain from the ISHE of Pt to the IEE of $\alpha$-Sn). One can say that proceeding from the ISHE of the heavy-metal layers to the IEE of a TI such as $\alpha$-Sn increases the conversion efficiency by about an order of magnitude. If we consider the gain in voltage for the same injected spin current density, the gain is even larger as, in most cases, the electrical resistance per square of a $2 D E G$ is higher than the corresponding resistance of a heavy-metal layer (see a similar discussion in the next paragraph for the gain in electrical power). 
TABLE I. Some examples of experimental data on spin-to-charge conversion coefficients $\lambda_{\mathrm{ISHE}}^{*}=\theta_{\mathrm{SHE}} \lambda_{\mathrm{SF}}$ for the ISHE of heavy metals (HM) and $\lambda_{\text {IEE }}$ for the IEE of 2D systems (topological insulators, Rashba interfaces). In order to compare the conversion efficiency of HMs and a TI such as $\alpha$-Sn, we present in the last column the ratios $\lambda_{\text {IEE }}(\alpha-\operatorname{Sn}) / \lambda_{\text {ISHE }}^{*}(\mathrm{HM})$ characterizing the gain in production of the charge current with the TI for the same injected spin current density. ${ }^{1}$ For Ta, we use the value of $\lambda_{\mathrm{SF}}$ given by Hahn et al. [24] to calculate two values of $\lambda_{\text {ISHE }}^{*}$ corresponding to the spin Hall angle data measured by Liu et al. [23] and Hahn et al. [24]. The position of the substrated is indicated by $/ /$.

\begin{tabular}{|c|c|c|c|c|c|c|c|}
\hline System & $\begin{array}{l}\text { Inverse Edelstein } \\
\text { length } \lambda_{\text {IEE }}(\mathrm{nm})\end{array}$ & $T(\mathrm{~K})$ & $\begin{array}{l}\text { Spin Hall } \\
\text { angle } \theta_{\mathrm{SHE}}\end{array}$ & $\begin{array}{l}\text { Spin diffusion } \\
\text { length } \lambda_{\mathrm{SF}} \\
(\mathrm{nm})\end{array}$ & $\begin{array}{c}\lambda_{\mathrm{ISHE}}^{*}= \\
\theta_{\mathrm{SHE}} \lambda_{\mathrm{SF}}(\mathrm{nm})\end{array}$ & $\begin{array}{l}\text { Gain } g \text { in } \\
\text { current pro } \\
\text { with IEE of } \\
\text { SHE } g=\end{array}$ & $\begin{array}{l}\text { charge } \\
\text { duction } \\
\text { f } \alpha-\mathrm{Sn} \text { Vs } \\
\left|\frac{\lambda_{\text {IIE }}^{(\alpha-S n)}}{\lambda_{\text {ISHE }}^{*}}\right|\end{array}$ \\
\hline $\begin{array}{l}/ / \mathrm{YIG} / \mathrm{Bi}_{2} \mathrm{Se}_{3} / \\
\mathrm{MgO}\end{array}$ & $0.035 \pm 0.004[13]$ & 300 & & & & & \\
\hline $\begin{array}{c}\mathrm{In}-\mathrm{Sb} / / \alpha-\mathrm{Sn} / \\
\mathrm{Ag} / \mathrm{Fe}\end{array}$ & $2.1 \pm 0.2[9]$ & 300 & & & & & \\
\hline $\mathrm{Bi} / \mathrm{Ag} / \mathrm{Ni}-\mathrm{Fe}$ & $0.2-0.3[5]$ & 300 & & & & & \\
\hline$[111] \mathrm{Ge} / / \mathrm{Fe}$ & $0.13[20]$ & 20 & & & & & \\
\hline $\begin{array}{l}\mathrm{SrTiO}_{3} / / \mathrm{LaAlO}_{3} / \\
\mathrm{Ni}-\mathrm{Fe} / \mathrm{Al}\end{array}$ & $6.4[10]$ & 7 & & & & & \\
\hline $\mathrm{Pt}$ & & 300 & $0.056 \pm 0.010[19]$ & $3.4 \pm 0.4[19]$ & 0.19 & 11. & \\
\hline \multirow[t]{2}{*}{ W } & & 300 & $-0.33 \pm 0.02[21]$ & Not given & & & \\
\hline & & 300 & $-0.27[22]$ & $1.26[22]$ & -0.34 & 6.2 & \\
\hline \multirow[t]{2}{*}{$\beta-\mathrm{Ta}^{1}$} & & 300 & $-0.15 \pm 0.03[23]$ & Not given & -0.27 & 7.8 & \\
\hline & & 300 & $-0.02 \pm_{0.015}^{0.008}[24]$ & $1.8 \pm 0.7[24]$ & -0.04 & 53 & \\
\hline
\end{tabular}

When the spin-to-charge conversion is obtained in spin pumping ferromagnetic resonance experiments, it is also interesting to compare directly the experimental charge current productions with heavy metals and 2DEGs for the same strength of radio-frequency magnetic field. Fig. 1 shows results on the maximum experimental production of the 2D charge current (current per unit of width) at room temperature by the ISHE of $\mathrm{Au}, \mathrm{AuW}, \mathrm{AuTa}$, and $\mathrm{Pt}$ and the IEE with a $\mathrm{Ag} / \mathrm{Bi}$ Rashba interface and $\alpha$-Sn TI. Although the ratio $I_{c}^{2 \mathrm{D}} /\left(h_{\mathrm{rf}}\right)^{2}$ is not exactly proportional to $j_{c}^{2 \mathrm{D}} / j_{s}^{3 \mathrm{D}}$ but also involves extrinsic parameters such the as interface spin mixing conductance $G^{\uparrow \downarrow}$, one sees that the experimental results on $I_{c}^{2 \mathrm{D}} /\left(h_{\mathrm{rf}}\right)^{2}$ follow rather well our calculations for $j_{c}^{2 \mathrm{D}} / j_{s}^{3 \mathrm{D}}$. In the data of Fig. 1, $\alpha$-Sn has a clear advantage and this advantage would be enhanced by normalizing the charge current production by the spin current injected.

In a device of the spin Seebeck power-generator type [27] based on the ISHE or IEE conversion of spin currents generated by thermal fluxes though a ferromagnetic layer (preferably insulating), we have to consider the gain in electrical power for the same injected spin current density. The production of electrical power scales with the product of the sheet resistance $r_{\square}$ of the SHE layer or 2DEG by the square of the total charge current $J_{C}$. Consequently, the advantage of IEE on ISHE comes not only from the more efficient conversion by the factor $\lambda_{\mathrm{IEE}} / \lambda_{\mathrm{ISHE}}^{*}$, but also from the higher resistance of the 2DEG. When one goes from ISHE (Pt, Ta, or W) to IEE ( $\alpha$-Sn), the gain in output power $P_{\text {out }}$ for the same injected spin current density can be written as

$$
\begin{aligned}
G & =\frac{P_{\text {out }}(\mathrm{IEE})}{P_{\mathrm{out}}(\mathrm{ISHE})}=\frac{r_{\square}(\mathrm{TI})}{r_{\square}(\mathrm{HM})} \times\left[\frac{j_{c}^{2 \mathrm{D}}(\mathrm{IEE})}{j_{c}^{2 \mathrm{D}}(\mathrm{ISHE})}\right]^{2} \\
& =\frac{r_{\square}(\mathrm{TI})}{r_{\square}(\mathrm{HM})} \times\left(\frac{\lambda_{\mathrm{IEE}}}{\lambda_{\mathrm{ISHE}}^{*}}\right)^{2} .
\end{aligned}
$$

To calculate the gain $G$, we took the values for $\lambda_{\text {IEE }}(\alpha-$ $\mathrm{Sn}) / \lambda_{\text {ISHE }}^{*}$ in Table I, the value $r_{\square}(\alpha-\mathrm{Sn}) \approx 16 \mathrm{k} \Omega$ estimated from the Fermi contour radius and mean free path for $\alpha$-Sn in [9], $r_{\square}(\mathrm{HM})=\rho / t$ derived for the thickness $t=\lambda_{\mathrm{SF}}$ giving an almost optimal efficiency of SHE, values of $\lambda_{\mathrm{SF}}$ in Table I, and the following experimental values of the resistivity: $\rho \approx 17.3 \times 10^{-8} \Omega \mathrm{m}$ for Pt [19], $\rho \approx 260$ $\times 10^{-8} \Omega \mathrm{m}$ for $\mathrm{W}$ [21], and $\rho \approx 190 \times 10^{-8} \Omega \mathrm{m}$ for Ta [23]. The result is that the large value of $r_{\square}$ (TI) for a 2DEG leads to very large gains for the production of electrical power from a spin current. From Eq. (9), the gain $G$ with the IEE of $\alpha$-Sn equals $3.8 \times 10^{4}, 295$, and 917 with respect to the energy production from SHE respectively in Pt, W, ( $\theta_{\mathrm{SHE}}$ from [21] and $\lambda_{\mathrm{SF}}$ from [22]), and $\mathrm{Ta}\left(\theta_{\mathrm{SHE}}\right.$ from [23] and $\lambda_{\mathrm{SF}}$ from [24]). However, we note that the above gains are calculated for the situation of an insulating magnetic layer (no shunt) and that the advantage of a large $r_{\square}$ (TI) disappears if there is shunting by a lowresistance metallic magnetic layer. We note that there are experimental evidences of the advantage of TI for the production of electrical power in spin-Seebeck-based devices. 


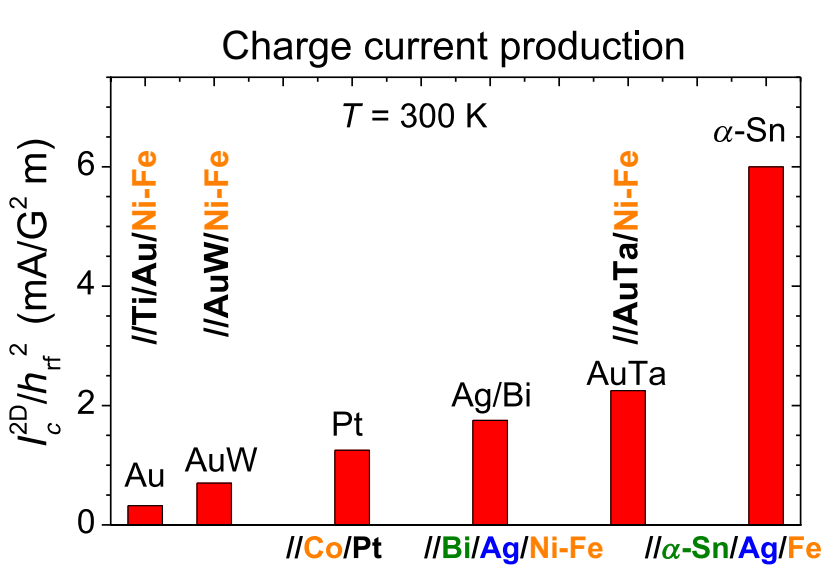

FIG. 1. Ratio between charge current production and $h_{\mathrm{rf}}^{2}$ (where $h_{\mathrm{rf}}$ is the magnetic rf field) by ISHE (Au [25], Pt [19], $\mathrm{Au}_{0.97} \mathrm{~W}_{0.07}$ [25], $\mathrm{Au}_{0.975} \mathrm{Ta}_{0.025}$ [26]) or IEE (Ag/Bi [5] and $\mathrm{Ag} / \alpha-\mathrm{Sn}[9])$ in experiments of spin pumping ferromagnetic resonance at room temperature. The results are obtained in the same setup $(f \sim 9.7 \mathrm{GHz})$ and with the same geometry (i.e., width of $0.4 \mathrm{~mm}$ ). The definitely larger experimental production with the $\alpha$-Sn TI is in reasonable agreement with the calculated gain results in Table I (if normalized by the injected spin current, the advantage of $\alpha$-Sn is even larger). We should point out that $I_{c}^{2 \mathrm{D}} / h_{\mathrm{rf}}^{2}$ depends not only on the intrinsic parameters of the spinorbit coupling material, $\theta_{\mathrm{SHE}}, \lambda_{\mathrm{SF}}, \lambda_{\mathrm{IEE}}$, but also on extrinsic parameters such as interface parameters throughout the whole stack.

For example, Jiang et al. has shown that the output voltage using $\left(\mathrm{Bi}_{0.24} \mathrm{Sb}_{0.76}\right)_{2} \mathrm{Te}_{3} / \mathrm{YIG}$ is 10 times the one using $\mathrm{Pt} / \mathrm{YIG}[28]$.

\section{CHARGE-TO-SPIN CURRENT CONVERSION: SPIN HALL EFFECT VS EDELSTEIN EFFECT}

The situation is a little more complex in the conversion of charge current to spin current and spin torque by a direct SHE or direct Edelstein effect in spin torque ferromagnetic resonance [12,29-33] or second harmonic technique experiments [34-40]. From the interpretation of the data, the experimentalist knows the spin current accommodated by the ferromagnet after coming from the heavy metal through the interface. Therefore, the interface transparency is also involved in the spin current accommodated by the adjacent ferromagnetic layer, for example, via the spin mixing conductance $G_{\uparrow \downarrow}$ [16]. For SHE, in the optimal conditions with transparent enough interfaces, the transferred spin current density $j_{s}^{3 \mathrm{D}}$ is simply related to the charge current $j_{c}^{3 \mathrm{D}}$ in the SHE layer by the expression $[30,41,42]$

$$
j_{s}^{3 \mathrm{D}}=\theta_{\mathrm{SHE}}\left(1-\operatorname{sech}\left[t / \lambda_{\mathrm{SF}}\right]\right) \times j_{c}^{3 \mathrm{D}}
$$

for a heavy metal of thickness $t$. With a total current or $2 \mathrm{D}$ charge current density $j_{c}^{2 \mathrm{D}}$ equal to $t \times j_{c}^{3 \mathrm{D}}$, this result leads to

$$
j_{s}^{3 \mathrm{D}}=\theta_{\mathrm{SHE}}\left(1-\operatorname{sech}\left[t / \lambda_{\mathrm{SF}}\right]\right) \frac{1}{t} \times j_{c}^{2 \mathrm{D}}
$$

and, for $t \cong 1.5 \lambda_{\mathrm{SF}}$, to a maximum ratio for the conversion from a $2 \mathrm{D}$ charge current density to a $3 \mathrm{D}$ spin current density equal to

$$
\frac{j_{s}^{3 \mathrm{D}}}{j_{c}^{2 \mathrm{D}}}=0.38 \frac{\theta_{\mathrm{SHE}}}{\lambda_{\mathrm{SF}}}=q^{*} .
$$

The maximum efficiency for the production of spin current density $\left(j_{s}^{3 \mathrm{D}}\right)$ and related spin torque density from a given $2 \mathrm{D}$ electrical current density $\left(j_{c}^{2 \mathrm{D}}\right)$ can be therefore characterized by the merit factor $q^{*}$, to be compared with the equivalent merit factor for conversion by the Edelstein effect in 2D systems, $q_{\mathrm{ICS}}$ in the notation of Kondou et al. [12], defined in Eq. (3).

When one goes from the SHE of heavy metal to the EE of TI, the gain $g^{\prime}$ in the 3D spin current density $j_{s}^{3 \mathrm{D}}$ and STT for the same $j_{c}^{2 \mathrm{D}}$ is

$$
g^{\prime}=\frac{q_{\mathrm{ICS}}}{q^{*}}=\frac{2.6 q_{\mathrm{ICS}} \lambda_{\mathrm{SF}}}{\theta_{\mathrm{SHE}}} .
$$

As the conversion ratios for the SHE and ISHE depend on the thickness $t$, the spin diffusion length $\lambda_{\mathrm{SF}}$ and the choice of the optimal $t / \lambda_{\mathrm{SF}}$ ratio (with different choices for spin-to-charge and charge-to-spin conversions), there is no strict symmetry but only similarities between Eq. (13) for the factor $g^{\prime}$ and Eq. (8) for the factor $g$ of the inverse conversion.

Calculating $q^{*}$ for $\mathrm{Pt}, \mathrm{W}$, and Ta from values of $\lambda_{\mathrm{SF}}$ and $\theta_{\text {SHE }}$ in Table I $[19,22-24]$ and taking as a reference the value $q_{\mathrm{ICS}} \approx 0.5 \mathrm{~nm}^{-1}$ found by Kondou et al. [12] for the TI $\left(\mathrm{Bi}_{1-\mathrm{x}} \mathrm{Sb}_{\mathrm{x}}\right)_{2} \mathrm{Te}_{3}$ for $x=0.5$ (0.5 turning out as the concentration $x$ with purely interface effects), we find that the gain $g^{\prime}$ in the production of spin current with the TI is 88.4 with respect to $\mathrm{Pt}, 6.1$ with respect to $\mathrm{W}$, and 15.6 with respect to $\mathrm{Ta}$ (calculation for Ta with $\theta_{\mathrm{SHE}}=0.15$ from [23] and $\lambda_{\mathrm{SF}}=1.8 \mathrm{~nm}$ from [24]). These gains are in the same range as the gains we find for the inverse conversion (however, note that the results on $g$ are based on the IEE results at room temperature on $\alpha$-Sn, whereas, for those on $g^{\prime}$, we took the EE results at low temperature for $\left(\mathrm{Bi}_{0.5} \mathrm{Sb}_{0.5}\right)_{2} \mathrm{Te}_{3}$.

\section{SHUNTING BY MAGNETIC METALLIC LAYER}

Finally, we consider the situation with a conducting magnetic layer of thickness $t_{F}$ and resistivity $\rho_{F}$ shunting the heavy metal or 2DEG (the shunt by the substrate or the bulk of the TI can be taken into account in the same way).

For the spin-to-charge conversion in both ISHE and IEE, there is no change in the production of the $2 \mathrm{D}$ charge current density $j_{c}^{2 \mathrm{D}}$ by a given injected spin current density 
$j_{s}^{3 \mathrm{D}}$ and the gain in current with IEE is still $\lambda_{\mathrm{IEE}} / \lambda_{\mathrm{ISHE}}^{*}[\mathrm{Eq}$. (8)], as calculated above (Table I).

In contrast, in the case of a spin Seebeck powergenerator type device, the production of electrical power is lowered by the reduction of the $2 \mathrm{D}$ resistances appearing in Eq. (9) by the respective factors $1 /\left(1+\rho_{\mathrm{HM}} t_{F} / \rho_{F} \lambda_{\mathrm{SF}}\right)$ for $r_{\square}(\mathrm{HM})$, when one assumes $t=\lambda_{\mathrm{SF}}$ for optimal conversion and by $1 /\left[1+r_{\square}(\mathrm{TI}) t_{F} / \rho_{F}\right]$ for $r_{\square}(\mathrm{TI})$. Thus, the gain $G$ of Eq. (9) can be rewritten, with shunting, as

$$
\begin{aligned}
G_{\mathrm{WS}}= & \frac{r_{\square}(\mathrm{TI})}{r_{\square}(\mathrm{HM})} \times\left(\frac{\lambda_{\mathrm{IEE}}}{\lambda_{\mathrm{ISHE}}^{*}}\right)^{2} \times \frac{1}{1+\left(\rho_{\mathrm{HM}} t_{F} / \rho_{F} \lambda_{\mathrm{SF}}\right)} \\
& \times \frac{1}{1+\left[r_{\square}(\mathrm{TI}) t_{F} / \rho_{F}\right]} .
\end{aligned}
$$

These reduction factors are the most important for heavy metals of large resistivity and short spin diffusion lengths (W, Ta), and even more for 2DEGs of large sheet resistance. With the parameters already used in the article for $\mathrm{Pt}, \mathrm{W}, \mathrm{Ta}$, or $\alpha$-Sn and typical values for the thickness of a metallic magnetic layer such as $\mathrm{CoFeB}, \rho_{F}=200 \mu \Omega \mathrm{cm}$, $t_{F}=5 \mathrm{~nm}$, we find that the gain $G_{\mathrm{WS}}$ in electrical power obtained by using the TI $\alpha-\mathrm{Sn}$ instead of Pt is reduced to about 807 . With respect to $\mathrm{W}, G_{\mathrm{WS}}$ with $\alpha$-Sn decreases to about 1.5 and, with respect to Ta, to about 6 or 55 , depending on the reference for the SHE or Ta. Shortly speaking, the advantage of the large 2D sheet resistance of the TI is partly compensated by dominant shunting effects.

It is straightforward to show that shunting by a metallic magnetic layer reduces the production of spin current from charge current (SHE or EE) by the same factors $1 /\left(1+\rho_{\mathrm{HM}} t_{F} / \rho_{F} \lambda_{\mathrm{SF}}\right)$ and $1 /\left[1+r_{\square}(\mathrm{TI}) t_{F} / \rho_{F}\right]$. By including the parameters used above and for the shunt by a metallic magnetic layer, we find that Pt, the heavy metal of lowest resistance and smallest shunting effects, gives the most efficient conversion. The situation would come back definitely at the advantage of a TI only if its sheet resistance amounts to only a few hundreds of ohms, and, of course, in the ideal situation with an insulating magnetic layer and no shunting effect. For instance, a relatively large IEE efficiency, $\lambda_{\text {IEE }}=0.4 \mathrm{~nm}$, has been reported using $\mathrm{YIG} / \mathrm{MoS}_{2}[43]$.

\section{SPIN HALL CONDUCTIVITY AND $q_{\text {ICS }}$}

The conversion between charge to spin currents in 2DEGs is not always described by $q_{\text {ICS }}$ but is also (correctly) characterized in some publications by the spin Hall conductivity $\sigma_{\mathrm{SH}}$, defined as the ratio between the spin current density $j_{S}$ and the electric field in the 2DEG and related to $q_{\text {ICS }}$ by

$$
\sigma_{\mathrm{SH}}=\frac{j_{s}^{3 \mathrm{D}}}{E_{x}}=q_{\mathrm{ICS}} \frac{j_{c}^{2 \mathrm{D}}}{E_{x}}=q_{\mathrm{ICS}} \sigma_{2 \mathrm{D}}
$$

where $\sigma_{2 \mathrm{D}}$ is the conductivity of the $2 \mathrm{DEG}$, so that $\sigma_{\mathrm{SH}}$ describes both the conversion from charge current to spin current (via $q_{\text {ICS }}$ ) and the conduction efficiency of the 2DEG (via $\sigma_{2 \mathrm{D}}$ ).

\section{3D PICTURE FOR 2DEGS}

In other publications, the $2 \mathrm{DEG}$ is treated as a layer of heavy metal characterized by its thickness $t$, a vertical spin diffusion length $\lambda_{\mathrm{SF}}$ uniformly distributed from the top to the bottom of the 2DEG, and a SHE angle $\theta_{\text {SHE }}$ also uniformly distributed. In spin-to-charge conversion (e.g., in spin pumping), the ratio $j_{c}^{2 \mathrm{D}} / j_{s}^{3 \mathrm{D}}$ is given by Eq. (6), $j_{c}^{2 \mathrm{D}} / j_{s}^{3 \mathrm{D}}=\theta_{\mathrm{SHE}} \lambda_{\mathrm{SF}} \tanh \left[t / 2 \lambda_{\mathrm{SF}}\right]$, and the result obtained for $\theta_{\text {SHE }}$ for the same experimental ratio depends on the choice of the thickness $t$ of the 2DEG and its vertical $\lambda_{\mathrm{SF}}$. Depending on this rather arbitrary choice, one finds a value of $\theta_{\text {SHE }}$ ranging between $2 j_{c}^{2 \mathrm{D}} /\left(j_{s}^{3 \mathrm{D}} t\right)$ and $j_{c}^{2 \mathrm{D}} /\left(j_{s}^{3 \mathrm{D}} \lambda_{s f}\right)$, i.e., expressed as a function of the intrinsic parameter $\lambda_{\text {IEE }}$ for conversion between $3 \mathrm{D}$ and $2 \mathrm{D}$ currents, between $2 \lambda_{\mathrm{IEE}} / t$ and $\lambda_{\mathrm{IEE}} / \lambda_{\mathrm{SF}}$. The arbitrary choice of the thickness and spin diffusion length of the 2DEG probably explains the large dispersion of the spin Hall angle obtained for similar TI. Similarly, for charge-to-spin conversion, the ratio $j_{s}^{3 \mathrm{D}} / j_{c}^{2 \mathrm{D}}$ is given by Eq. $(11), j_{s}^{3 \mathrm{D}}=$ $\theta_{\mathrm{SHE}}\left(1-\operatorname{sech}\left[t / \lambda_{\mathrm{SF}}\right]\right) \frac{1}{t} \times j_{c}^{2 \mathrm{D}}$. Thus, the same experimental value of $j_{s}^{3 \mathrm{D}} / j_{c}^{2 \mathrm{D}}$ leads to values of $\theta_{\mathrm{SHE}}$ depending on the choice of $t$ and $\lambda_{\mathrm{SF}}$. For example, it results in $\left(j_{s}^{3 \mathrm{D}} / j_{c}^{2 \mathrm{D}}\right)\left(2 \lambda_{\mathrm{SF}}^{2} / t\right)$ for $t \ll 1.5 \lambda_{\mathrm{SF}}$ and $\left(j_{s}^{3 \mathrm{D}} / j_{c}^{2 \mathrm{D}}\right)\left(2.6 \lambda_{\mathrm{SF}}\right)$ when $t \cong 1.5 \lambda_{\mathrm{SF}}$. This arbitrary choice, again, can explain the dispersion of the spin Hall angle derived from similar experimental values.

\section{CONCLUSIONS}

The objective of this article is to clarify the problem of the parametrization of spin-to-charge conversion experiments by SOC. We have proposed a way to compare the efficiency of the conversion in 3D and 2D systems when they are characterized by the spin Hall angle $\theta_{\text {SHE }}$ for the SHE of 3D heavy metals and the parameters $\lambda_{\text {IEE }}$ or $q_{\text {ICS }}$ for the inverse Edelstein or Edelstein effects in 2DEGs. By considering some examples of heavy metals and topological insulators, we find that, in these examples, the production of charge current from spin current and spin current by charge current is more efficient by about 1 order of magnitude with the 2DEGs of topological insulators.

The higher $r_{\square}$ (resistance per square) in 2DEGs means that the advantage of the TI is even more important when considering the production of voltage (as in the magnetoelectric spin-orbit logic device proposed by Intel [44]) or electrical power (as in devices such as a spin Seebeck power generator [27]). With a shunt by typical metallic magnetic layers, the large advantage of TI in terms of charge current production from spin current remains. In 
contrast, in terms of spin current production from charge current, the advantage is expected to be with HMs of low resistivity such as $\mathrm{Pt}$ or $2 \mathrm{DEGs}$ of resistance per square below $1 \mathrm{k} \Omega$.

[1] I. L. Prejbeanu, S. Bandiera, J. Alvarez-Hérault, R. C. Sousa, B. Dieny, and J.-P. Nozières, Thermally assisted MRAMs: Ultimate scalability and logic functionalities, J. Phys. D. Appl. Phys. 46, 74002 (2013).

[2] Z. Wang, W. Zhao, E. Deng, J. Klein, and C. Chappert, Perpendicular-anisotropy magnetic tunnel junction switched by spin-Hall-assisted spin-transfer torque, J. Phys. D. Appl. Phys. 48, 065001 (2015).

[3] A. Hoffmann, Spin Hall effects in metals, IEEE Trans. Magn. 49, 5172 (2013).

[4] V. M. Edelstein, Spin polarization of conduction electrons induced by electric current in two-dimensional asymmetric electron systems, Solid State Commun. 73, 233 (1990).

[5] J. C. Rojas Sánchez, L. Vila, G. Desfonds, S. Gambarelli, J. P. Attané, J. M. De Teresa, C. Magén, and A. Fert, Spin-tocharge conversion using Rashba coupling at the interface between non-magnetic materials, Nat. Commun. 4, 2944 (2013).

[6] Y. Shiomi, K. Nomura, Y. Kajiwara, K. Eto, M. Novak, K. Segawa, Y. Ando, and E. Saitoh, Spin-Electricity Conversion Induced by Spin Injection into Topological Insulators, Phys. Rev. Lett. 113, 196601 (2014).

[7] A. R. Mellnik, J. S. Lee, A. Richardella, J. L. Grab, P. J. Mintun, M. H. Fischer, A. Vaezi, A. Manchon, E.-A. Kim, N. Samarth, and D. C. Ralph, Spin-transfer torque generated by a topological insulator, Nature 511, 449 (2014).

[8] A. Nomura, T. Tashiro, H. Nakayama, and K. Ando, Temperature dependence of inverse Rashba-Edelstein effect at metallic interface, Appl. Phys. Lett. 106, 212403 (2015).

[9] J. C. Rojas-Sánchez, S. Oyarzún, Y. Fu, A. Marty, C. Vergnaud, S. Gambarelli, L. Vila, M. Jamet, Y. Ohtsubo, A. Taleb-Ibrahimi, P. Le Fèvre, F. Bertran, N. Reyren, J. M. George, and A. Fert, Spin to Charge Conversion at Room Temperature by Spin Pumping into a New Type of Topological Insulator: $\alpha$-Sn Films, Phys. Rev. Lett. 116, 096602 (2016).

[10] E. Lesne, Y. Fu, S. Oyarzún, J. C. Rojas Sánchez, D. C. Vaz, H. Naganuma, G. Siccoli, J.-P. Attané, M. Jamet, J.-M. George, A. Barthélémy, H. Jaffrès, A. Fert, M. Bibes, and L. Vila, Highly efficient and tunable spin-to-charge conversion through Rashba coupling at oxide interfaces, Nat. Mater. 15, 1261 (2016).

[11] C. H. Li, O. M. J. van 't Erve, J. T. Robinson, Y. Liu, L. Li, and B. T. Jonker, Electrical detection of charge-currentinduced spin polarization due to spin-momentum locking in Bi2Se3, Nat. Nanotechnol. 9, 218 (2014).

[12] K. Kondou, R. Yoshimi, A. Tsukazaki, Y. Fukuma, J. Matsuno, K. S. Takahashi, M. Kawasaki, Y. Tokura, and Y. Otani, Fermi level dependent charge-to-spin current conversion by Dirac surface state of topological insulators, Nat. Phys. 1510, 1027 (2016).
[13] H. Wang, J. Kally, J. S. Lee, T. Liu, H. Chang, D. R. Hickey, K. A. Mkhoyan, M. Wu, A. Richardella, and N. Samarth, Surface-State-Dominated Spin-Charge Current Conversion in Topological-Insulator-Ferromagnetic-Insulator Heterostructures, Phys. Rev. Lett. 117, 076601 (2016).

[14] Y. Ando and M. Shiraishi, Spin to charge interconversion phenomena in the interface and surface states, J. Phys. Soc. Japan 86, 011001 (2017).

[15] A. Soumyanarayanan, N. Reyren, A. Fert, and C. Panagopoulos, Review Emergent phenomena induced by spin - orbit coupling at surfaces and interfaces, Nature 539, 509 (2016).

[16] S. Zhang and A. Fert, Conversion between spin and charge currents with topological insulators, Phys. Rev. B 94, 184423 (2016).

[17] A. Azevedo, L. H. Vilela-Leão, R. L. Rodríguez-Suárez, A. F. Lacerda Santos, and S. M. Rezende, Spin pumping and anisotropic magnetoresistance voltages in magnetic bilayers: Theory and experiment, Phys. Rev. B 83, 144402 (2011).

[18] Y. Tserkovnyak, A. Brataas, G. E. W. Bauer, and B. I. Halperin, Nonlocal magnetization dynamics in ferromagnetic heterostructures, Rev. Mod. Phys. 77, 1375 (2005).

[19] J.-C. Rojas-Sánchez, N. Reyren, P. Laczkowski, W. Savero, J.-P. Attané, C. Deranlot, M. Jamet, J.-M. George, L. Vila, and H. Jaffrès, Spin Pumping and Inverse Spin Hall Effect in Platinum: The Essential Role of Spin-Memory Loss at Metallic Interfaces, Phys. Rev. Lett. 112, 106602 (2014).

[20] S. Oyarzún, A. K. Nandy, F. Rortais, J.-C. Rojas-Sánchez, M. T. Dau, and P. Noël, Evidence for spin-to-charge conversion by Rashba coupling in metallic states at the Fe/Ge(111) interface, Nat. Commun. 7, 13857 (2016).

[21] C.-F. Pai, L. Liu, Y. Li, H. W. Tseng, D. C. Ralph, and R. A. Buhrman, Spin transfer torque devices utilizing the giant spin Hall effect of tungsten, Appl. Phys. Lett. 101, 122404 (2012).

[22] J. Kim, P. Sheng, S. Takahashi, S. Mitani, and M. Hayashi, Spin Hall Magnetoresistance in Metallic Bilayers, Phys. Rev. Lett. 116, 097201 (2016).

[23] L. Liu, C.-F. Pai, Y. Li, H. W. Tseng, D. C. Ralph, and R. A. Buhrman, Spin-torque switching with the giant spin Hall effect of tantalum, Science 336, 555 (2012).

[24] C. Hahn, G. de Loubens, O. Klein, M. Viret, V. V. Naletov, and J. Ben Youssef, Comparative measurements of inverse spin Hall effects and magnetoresistance in YIG/Pt and YIG/Ta, Phys. Rev. B 87, 174417 (2013).

[25] P. Laczkowski, J. C. Rojas-Sánchez, W. Savero-Torres, H. Jaffrès, N. Reyren, C. Deranlot, L. Notin, C. Beigné, A. Marty, J. P. Attané, L. Vila, J. M. George, and A. Fert, Experimental evidences of a large extrinsic spin Hall effect in AuW alloy, Appl. Phys. Lett. 104, 142403 (2014).

[26] P. Laczkowski, Y. Fu, H. Yang, J.-C. Rojas-Sánchez, P. Noel, V. T. Pham, G. Zahnd, C. Deranlot, S. Collin, C. Bouard, P. Warin, V. Maurel, M. Chshiev, A. Marty, J.-P. Attané, A. Fert, H. Jaffrès, L. Vila, and J.-M. George, Large enhancement of the spin Hall effect in Au by side-jump scattering on Ta impurities, Phys. Rev. B 96, 140405(R) (2017). 
[27] K. ichi Uchida, H. Adachi, T. Kikkawa, A. Kirihara, M. Ishida, S. Yorozu, S. Maekawa, and E. Saitoh, Thermoelectric generation based on spin seebeck effects, Proc. IEEE 104, 1946 (2016).

[28] Z. Jiang, C. Chang, M. R. Masir, C. Tang, Y. Xu, J. S. Moodera, A. H. MacDonald, and J. Shi, Enhanced spin Seebeck effect signal due to spin-momentum locked topological surface states, Nat. Commun. 7, 11458 (2016).

[29] D. Fang, H. Kurebayashi, J. Wunderlich, K. Vyborny, R. P. Zarbo, R. P. Campion, A. Casiraghi, B. L. Gallagher, T. Jungwirth, and A. J. Ferguson, Spin - orbitdriven ferromagnetic resonance, Nat Nanotechnol. 6, 413 (2011).

[30] L. Liu, T. Moriyama, D. C. Ralph, and R. A. Buhrman, Spin-Torque Ferromagnetic Resonance Induced by the Spin Hall Effect, Phys. Rev. Lett. 106, 036601 (2011).

[31] K. Kondou, H. Sukegawa, S. Mitani, K. Tsukagoshi, and S. Kasai, Evaluation of spin Hall angle and spin diffusion length by using spin current-induced ferromagnetic resonance, Appl. Phys. Express 5, 1 (2012).

[32] A. Ganguly, K. Kondou, H. Sukegawa, S. Mitani, S. Kasai, Y. Niimi, Y. Otani, and A. Barman, Thickness dependence of spin torque ferromagnetic resonance in $\mathrm{Co} 75 \mathrm{Fe} 25 / \mathrm{Pt}$ bilayer films, Appl. Phys. Lett. 104, 072405 (2014).

[33] C. Guillemard, S. Petit-Watelot, S. Andrieu, and J.-C. Rojas-Sánchez, Charge-spin current conversion in high quality epitaxial $\mathrm{Fe} / \mathrm{Pt}$ systems: Isotropic spin Hall angle along different in-plane crystalline directions, Appl. Phys. Lett. 113, 262404 (2018).

[34] J. Kim, J. Sinha, M. Hayashi, M. Yamanouchi, S. Fukami, T. Suzuki, S. Mitani, and H. Ohno, Layer thickness dependence of the current-induced effective field vector in $\mathrm{Ta}|\mathrm{CoFeB}| \mathrm{MgO}$, Nat. Mater. 12, 240 (2012).

[35] M. Hayashi, J. Kim, M. Yamanouchi, and H. Ohno, Quantitative characterization of the spin-orbit torque using harmonic Hall voltage measurements, Phys. Rev. B 89, 144425 (2014).
[36] J. Torrejon, J. Kim, J. Sinha, S. Mitani, M. Hayashi, M. Yamanouchi, and $\mathrm{H}$. Ohno, Interface control of the magnetic chirality in $\mathrm{CoFeB} / \mathrm{MgO}$ heterostructures with heavy-metal underlayers, Nat. Commun. 5, 4655 (2014).

[37] K. Garello, I. M. Miron, C. O. Avci, F. Freimuth, Y. Mokrousov, S. Blügel, S. Auffret, O. Boulle, G. Gaudin, and P. Gambardella, Symmetry and magnitude of spin-orbit torques in ferromagnetic heterostructures, Nat. Nanotechnol. 8, 587 (2013).

[38] C. O. Avci, K. Garello, M. Gabureac, A. Ghosh, A. Fuhrer, S. F. Alvarado, and P. Gambardella, Interplay of spin-orbit torque and thermoelectric effects in ferromagnet/normalmetal bilayers, Phys. Rev. B 90, 224427 (2014).

[39] K. Ueda, M. Mann, C. Pai, A. Tan, G. S. D. Beach, K. Ueda, M. Mann, C. Pai, A. Tan, and G. S. D. Beach, Spin-orbit torques in $\mathrm{Ta} / \mathrm{TbxCo} 100-\mathrm{x}$ ferrimagnetic alloy films with bulk perpendicular magnetic anisotropy, Appl. Phys. Lett. 109, 232403 (2016).

[40] Y. Chen, D. Roy, E. Cogulu, H. Chang, M. Wu, and A. D. Kent, First harmonic measurements of the spin Seebeck effect 1, Appl. Phys. Lett. 113, 202403 (2018).

[41] P. C. van Son, H. van Kempen, and P. Wyder, Boundary Resistance of the Ferromagnetic-Nonferromagnetic Metal Interface, Phys. Rev. Lett. 58, 2271 (1987).

[42] J. Kim, J. Sinha, S. Mitani, M. Hayashi, S. Takahashi, S. Maekawa, M. Yamanouchi, and H. Ohno, Anomalous temperature dependence of current-induced torques in $\mathrm{CoFeB} / \mathrm{MgO}$ heterostructures with Ta-based underlayers, Phys. Rev. B 89, 174424 (2014).

[43] J. B. S. Mendes, A. Aparecido-Ferreira, J. Holanda, A. Azevedo, and S. M. Rezende, Efficient spin to charge current conversion in the $2 \mathrm{D}$ semiconductor $\mathrm{MoS}_{2}$ by spin pumping from yttrium iron garnet, Appl. Phys. Lett. 112, 242407 (2018).

[44] S. Manipatruni, D. E. Nikonov, C. Lin, T. A. Gosavi, H. Liu, B. Prasad, Y. Huang, E. Bonturim, R. Ramesh, and I. A. Young, Scalable energy-efficient magnetoelectric spin-orbit logic, Nature 565, 35 (2019). 